INPLASY

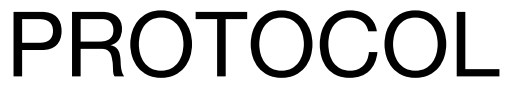

To cite: Yang et al.

Comparisons of therapeutic

safety of seven oral antimuscarinic drugs in

patients with overactive

bladder: A network meta-

analysis. Inplasy protocol

202170095. doi:

10.37766/inplasy2021.7.0095

Received: 30 July 2021

Published: 30 July 2021

Corresponding author:

Zhang Xiaopeng

xiaopengzj@126.com

Author Affiliation:

Xinchang People's Hospital

Support: No financial support.

Review Stage at time of this submission: Completed but not published.

Conflicts of interest:

None declared.

\section{Comparisons of therapeutic safety of seven oral antimuscarinic drugs in patients with overactive bladder: A network meta-analysis}

Yang, NN1; WU, QY2; Xu, FR3; Zhang, XP4.

Review question / Objective: P: patients with overactive bladder; I: patients were treated with medication; C: seven commonly used oral antimuscarinic drugs (darifenacin, fesoterodine, imidafenacin, oxybutynin, propiverine, solifenacin, tolterodine); 0 : Our findings suggest that tolterodine may be preferable in several AEs, as has higher probabilities of less being associated with important adverse events. Darifenacin, fesoterodine, imidafenacin, oxybutynin, and solifenacin have similar safety profiles in treating $O A B$ patients. Taken together, this analysis would be valuable for giving a grasp overview of the therapeutic safety for oral antimuscarinic drugs and be useful for personalized medicine for OAB patients.

Condition being studied: Antimuscarinic drugs for treating overactive bladder with adverse events. PubMed, the Cochrane Library, EMBASE, CNKI, Wanfang were searched for randomized controlled trials (RCTs). Studies comparing one or more antimuscarinic drugs for treating $O A B$ with adverse events reported were eligible. Data were extracted and analyzed with a network meta-analysis by two authors independently.

INPLASY registration number: This protocol was registered with the International Platform of Registered Systematic Review and Meta-Analysis Protocols (INPLASY) on 30 July 2021 and was last updated on 30 July 2021 (registration number INPLASY202170095).

\section{INTRODUCTION}

Review question / Objective: P: patients with overactive bladder; I: patients were treated with medication; C: seven commonly used oral antimuscarinic drugs (darifenacin, fesoterodine, imidafenacin, oxybutynin, propiverine, solifenacin, tolterodine); $O$ : Our findings suggest that tolterodine may be preferable in several AEs, as has higher probabilities of less being associated with important adverse events. Darifenacin, fesoterodine, imidafenacin, oxybutynin, and solifenacin 
have similar safety profiles in treating $O A B$ patients. Taken together, this analysis would be valuable for giving a grasp overview of the therapeutic safety for oral antimuscarinic drugs and be useful for personalized medicine for OAB patients.

Condition being studied: Antimuscarinic drugs for treating overactive bladder with adverse events. PubMed, the Cochrane Library, EMBASE, CNKI, Wanfang were searched for randomized controlled trials (RCTs). Studies comparing one or more antimuscarinic drugs for treating $O A B$ with adverse events reported were eligible. Data were extracted and analyzed with a network meta-analysis by two authors independently.

\section{METHODS}

Participant or population: P: patients with overactive bladder (45 eligible trials with 124587 patients).

Intervention: Patients were treated with antimuscarinic drugs such as darifenacin, fesoterodine, imidafenacin, oxybutynin, propiverine, solifenacin, and tolterodine Patients were treated with an antimuscarinic drug or placebo or other antimuscarinic drug.

Comparator: Patients were treated with placebo or other antimuscarinic drugs.

Study designs to be included: This study was a meta-analysis and reported safety outcomes such as xerostomia, constipation, urinary retention and blurred vision. This study did not include reviews, meta-analyses, observational studies, and preliminary studies, but allowed multigroup studies with no restrictions on the gender of patients and the cause of overactive bladderdiabetes.

Eligibility criteria: The inclusion criteria were as follows: (1) the study had a randomized clinical trials (RCT) design and compared an antimuscarinic drug with placebo or other antimuscarinic drugs in patients diagnosed as OAB; (2) the included antimuscarinic drugs were darifenacin, fesoterodine, imidafenacin, oxybutynin, propiverine, solifenacin, and tolterodine; (3) safety outcomes, such as dry mouth, constipation, urinary retention, blurred vision, etc. were reported in the study. Reviews, meta-analyses, observational studies, and pilot studies were excluded. Multi-arm studies were allowed. No restrictions on the sex of the patients or the cause of the OAB.

Information sources: The following electronic database was searched (from database inception until March 2020): PubMed, the Cochrane Library, Embase, CNKI, and Wangfang with only Chinese and English papers were accepted. The following keywords were included: "overactive bladder", "urinary incontinence", "darifenacin", "fes oterodine", "imidafenacin", "oxybutynin", "propiverine", "solifenacin", and "tolterodine".

Main outcome(s): Forty-five RCTs and 124587 patients were included. Results demonstrated that tolterodine had better safety outcomes in 7 out of 12 major adverse effects(AEs), including dry mouth, constipation, urinary retention, dizziness, urinary tract infection, dry eyes, and dry skin. Darifenacin, fesoterodine, imidafenacin, oxybutynin, and solifenacin presented comparable safety profiles with each other.

Quality assessment / Risk of bias analysis: All data were assessed by 2 investigators independently and disagreements were resolved by a third author. Baseline characteristics (sample size, age, and intervention), outcome data, and quality information were extracted from eligible studies. Data analysis was conducted with Stata 15.0 (StataCorp LLC, College Station, Texas, USA). The results are presented as standard mean differences (SMDs) and $95 \% \mathrm{Cl}$. In order to obtain the differences between each of the treatments with direct and indirect evidence, a network metaanalysis in a frequentist framework was used to analyze each outcome. $P$ values < 0.05 were considered statistically significant. A ranking for the treatments 
was produced for each outcome. Inconsistency was assessed by calculating the inconsistency factors.

Strategy of data synthesis: A total of 45 articles were selected from 248 articles based on the inclusion and exclusion criteria detailed in Methods. This resulted in 45 eligible trials with 124587 patients. Ten studies were performed in female patients only, accounting for $22 \%$ of the total studies. Twelve weeks was the most common length of treatment $(18 / 45,40 \%)$. Five studies were shorter than 4 weeks, 13 were between 4-12 weeks, and 9 were longer than 12 weeks. Thirty-two studies $(71 \%)$ used fixed doses during the trial. The safety outcomes were extracted from the eligible studies and used for network metaanalysis. The evaluated safety outcomes including dry mouth, constipation, urinary retention, blurred vision, dizziness, headache, urinary tract infection, dry eyes, dry skin, abdominal pain tenderness, dyspepsia, and dysuria. The results are detailed as follows.

Subgroup analysis: No subgroup analysis was performed.

Sensitivity analysis: No sensitivity analysis was performed.

Country(ies) involved: China.

Keywords: Antimuscarinic drugs, Network meta-analysis, Overactive bladder, Tolterodine, Systematic review.

Contributions of each author:

Author 1 - Yang Nannan.

Author 2 - Wu Qiaoyan.

Author 3 - Xu Faren.

Author 4 - Zhang Xiaopeng. 